\title{
A new procedure to analyze angular correlation experimental data
}

\author{
Guilherme S. Zahn ${ }^{1, a}$, Frederico A. Genezini ${ }^{2}$, Cibele B. Zamboni ${ }^{1}$, and Manoel T.F. da Cruz ${ }^{3}$ \\ ${ }^{1}$ Instituto de Pesquisas Energéticas e Nucleares, CP 11049, 05422-970 São Paulo, SP, Brazil \\ 2 Centro Regional de Ciências Nucleares - CRCN-CNEN/PE, Brazil \\ 3 Instituto de Física da Universidade de São Paulo, São Paulo, Brazil
}

\begin{abstract}
In this work an approach is proposed to the analysis of directional angular correlation data in which the function $W(\theta)$ used to fit the experimental data already includes the relations between the $A_{k k}$ parameters and the mixing ratios $(\delta)$, so that the angular correlation function is fitted explicitly in terms of the mixing ratio of the transition of interest; the normalization factor that arises from the fit can also be used, under certain situations, to determine the intensity of one of the transitions. In order to verify the usability and consistence of this method, some well-determined cascades from ${ }^{152} \mathrm{Eu}$ and ${ }^{60} \mathrm{Co}$ standard sources, as well as from the $\beta^{-}$decay of ${ }^{193} \mathrm{Os}$, were analyzed, and the results compared to the literature values, allowing for a discussion of the performance of this approach.
\end{abstract}

\section{Introduction}

Directional $\gamma \gamma$ angular correlation experiments have been widely used to measure nuclear multipolar mixing ratios $\left(\delta_{L, L+1}\right)$ for more than 40 years [1], and has the ability to measure not only the magnitude but also the signal of the mixing ratio.

The basic concept behind these measurements is that, in a sample where nuclear spins are randomly aligned, once you choose nuclei that emmitted a photon $\gamma_{1}$ in a direction $\hat{x}_{1}$, if there is a second transition $\gamma_{2}$ emmitted subsequently by the same nucleus, the direction $\hat{x}_{2}$ of this emmission is related to the nature and multipolarity of both transitions.

Strictly speaking, if a nuclear state decays by the successive emmission of two gamma transitions $\gamma_{1}$ and $\gamma_{2}$, which are in turn detected by two finite detectors which make an angle $\theta$ between their axes, the count rate will follow the angular correlation function [1], where the series was truncated to the order of $k=4$ due to experimental sensitivity limitations:

$$
W(\theta)=\alpha\left[1+A_{22} Q_{22} P_{2}(\cos \theta)+A_{44} Q_{44} P_{4}(\cos \theta)\right]
$$

where $A_{k k}$ are the angular correlation coefficients related to the properties of the transitions, $P_{k}$ are the Legendre Polynomials of the $k^{\text {th }}$ order, $Q_{k k}$ are coefficientes related to finite solid angle corrections (see [2,3]) and $\alpha$ is a normalization constant.

The $A_{k k}$ coefficients can be split in two separate coefficients, each related to one of the transitions involved:

$$
A_{k k}=A_{k}\left(\gamma_{1}\right) \cdot A_{k}\left(\gamma_{2}\right)
$$

and these coefficients can be expressed as:

$$
\begin{aligned}
A_{k}\left(\gamma_{1}\right)= & {\left[1+\delta^{2}\left(\gamma_{1}\right)\right]^{-1} \times\left[F_{k}\left(L_{1} L_{1} I_{i} I\right)+\right.} \\
& \left.+(-1)^{L_{1}+L_{1}^{\prime}} 2 \delta\left(\gamma_{1}\right) F_{k}\left(L_{1} L_{1}^{\prime} I_{i} I\right)+\delta^{2}\left(\gamma_{1}\right) F_{k}\left(L_{1}^{\prime} L_{1}^{\prime} I_{i} I\right)\right]
\end{aligned}
$$

and

$$
\begin{aligned}
A_{k}\left(\gamma_{2}\right)= & {\left[1+\delta^{2}\left(\gamma_{2}\right)\right]^{-1} \times\left[F_{k}\left(L_{2} L_{2} I_{f} I\right)+\right.} \\
& \left.+2 \delta\left(\gamma_{2}\right) F_{k}\left(L_{2} L_{2}^{\prime} I_{f} I\right)+\delta^{2}\left(\gamma_{2}\right) F_{k}\left(L_{2}^{\prime} L_{2}^{\prime} I_{f} I\right)\right]
\end{aligned}
$$

\footnotetext{
${ }^{a}$ Presenting author, e-mail: gzahn@ipen.br
}

where the $F_{k}$ are the Frauenfelder coefficients (tabulated in [4]), $L$ is the angular momentum carried away by the transition, $L^{\prime}=L+1$, and $\delta$ is the multipolar mixing ratio, obtained by the ratio of the reduced matrix elements of the multipolar electromagnectical transitions of the order $L$ e $L^{\prime}$.

\section{Fitting the angular correlation function}

The usual way to fit the experimental points to the angular correlation function $W(\theta)$ is to fit directly the three parameters that appear in equation (1) $\left(\alpha, A_{22}\right.$ and $\left.A_{44}\right)$ without taking into account the constraints between the latter two and the multipolar mixing ratio $(\delta)$, which can be seen in equations (3) and (4); in this fit all the data can be normalized in respect to a certain angle, thus eliminating the normalization constant $\alpha$ - introducing, as a side effect, some correlation between the data - and then finding the value of $\delta$ which minimizes the $\chi^{2}$.

In this work, a different approach will be taken, fitting the angular correlation function directly as a function of the parameters $\alpha$ and $\delta$. The main difference between the two procedures can be understood by noticing that, although equations 3 and 4 impose a constraint between the values of $A_{22}$ and $A_{44}$, only the second procedure effectively guarantees that this constraint will be respected.

Formally, this procedure can be described as follows; the equation to be fitted is:

$$
W_{\text {teo }}=\left(\frac{S \cdot Y_{12}}{4}\right) \cdot \epsilon \cdot R \cdot X
$$

where $S$ is the number of disintegrations in the sample in a time interval $\Delta t, Y_{12}$ is the probability that the pair of transitions $\gamma_{1} \gamma_{2}$ will occur per disintegration, and $\epsilon$ is the efficiency matrix (a diagonal $N \times N$ matrix, where $N$ is the number of independent detector pairs); $\epsilon, R$ and $X$ are defined as follows. If $J_{0}^{k}\left(E_{l}\right)$ is the absolute gamma ray efficiency of the detector $k$ to the energy $E_{l}$, then the efficiency matrix is 
given by:

$$
\epsilon=\left(\begin{array}{ccc}
J_{0}^{a}\left(E_{1}\right) \cdot J_{0}^{b}\left(E_{2}\right) & \cdots & 0 \\
\vdots & \ddots & \vdots \\
0 & \cdots & J_{0}^{c}\left(E_{1}\right) \cdot J_{0}^{d}\left(E_{2}\right)
\end{array}\right) .
$$

Moreover, if $Q_{k k}^{i j}$ is the $k^{t h}$ order solid angle correction factor (as defined in $[2,3]$ ) for the detector pair $i j$, then

$$
R=\left(\begin{array}{ccc}
1 & P_{2}\left(\cos \theta_{i j}\right) \cdot Q_{22}^{i j} & P_{4}\left(\cos \theta_{i j}\right) \cdot Q_{44}^{i j} \\
\vdots & \ddots & \vdots \\
1 & P_{2}\left(\cos \theta_{i j}\right) \cdot Q_{22}^{i j} & P_{4}\left(\cos \theta_{i j}\right) \cdot Q_{44}^{i j}
\end{array}\right)
$$

and, finally,

$$
X=\left(\begin{array}{c}
1 \\
A_{22}\left(\delta_{1}, \delta_{2}\right) \\
A_{44}\left(\delta_{1}, \delta_{2}\right)
\end{array}\right) .
$$

The parameter to be minimized is, then,

$$
\chi^{2}=\left(W_{\text {exp }}-W_{\text {teo }}\right)^{t} \cdot M^{-1} \cdot\left(W_{\text {exp }}-W_{\text {teo }}\right)
$$

where $M$ is the covariance matrix:

$$
M=M_{e x p}+M_{\epsilon} .
$$

\section{Experimental procedure and data analysis}

In order to test the proposed method, the values of the multipole mixing ratio for several transitions from standard sources of ${ }^{152} \mathrm{Eu}$ and ${ }^{60} \mathrm{Co}$, as well as transitions from the $\beta^{-}$ decay of ${ }^{193} \mathrm{Os}$, were measured and compared to the compiled values $[5,6]$.

These experimental measurements were performed using the planar multidetector array system assembled at the Laboratrio do Acelerador Linear (LAL), in the Physics Institute of the São Paulo University [7]. For this experiment, four HPGe detectors, with volumes ranging from 50 to $120 \mathrm{~cm}^{3}$, were used in coincidence mode, placed around the sample. The coincidence electronics is a regular fast-slow system that checks for a coincidence between two or more detectors within $200 \mathrm{~ns}$ and, for each valid event, stores both time and energy information for each detector involved. Both the detector setup and the electronics are shown in figure 1 .

The ${ }^{193}$ Os samples were produced by irradiating $5 \mathrm{mg}$ of $99 \%$ enriched ${ }^{192}$ Os for 5 minutes in the IEA-R1 reactor, under a neutron flux of about $10^{12} \mathrm{~cm}^{-2} \cdot \mathrm{s}^{-1}$, resulting in a total of $\sim 10^{8}$ total events; for the standard sources of ${ }^{60} \mathrm{Co}$ and ${ }^{152} \mathrm{Eu}$ the total number of events was approximately $10^{5}$ and $10^{6}$, respectively.

The data analysis was performed subtracting accidental coincidences, through the use of a time gate, and fitting bidimensional gaussian peaks compensated for Compton remains of other transitions.

The data fitting procedure was performed using a covariant fitting procedure developed in the MatLab platform, and

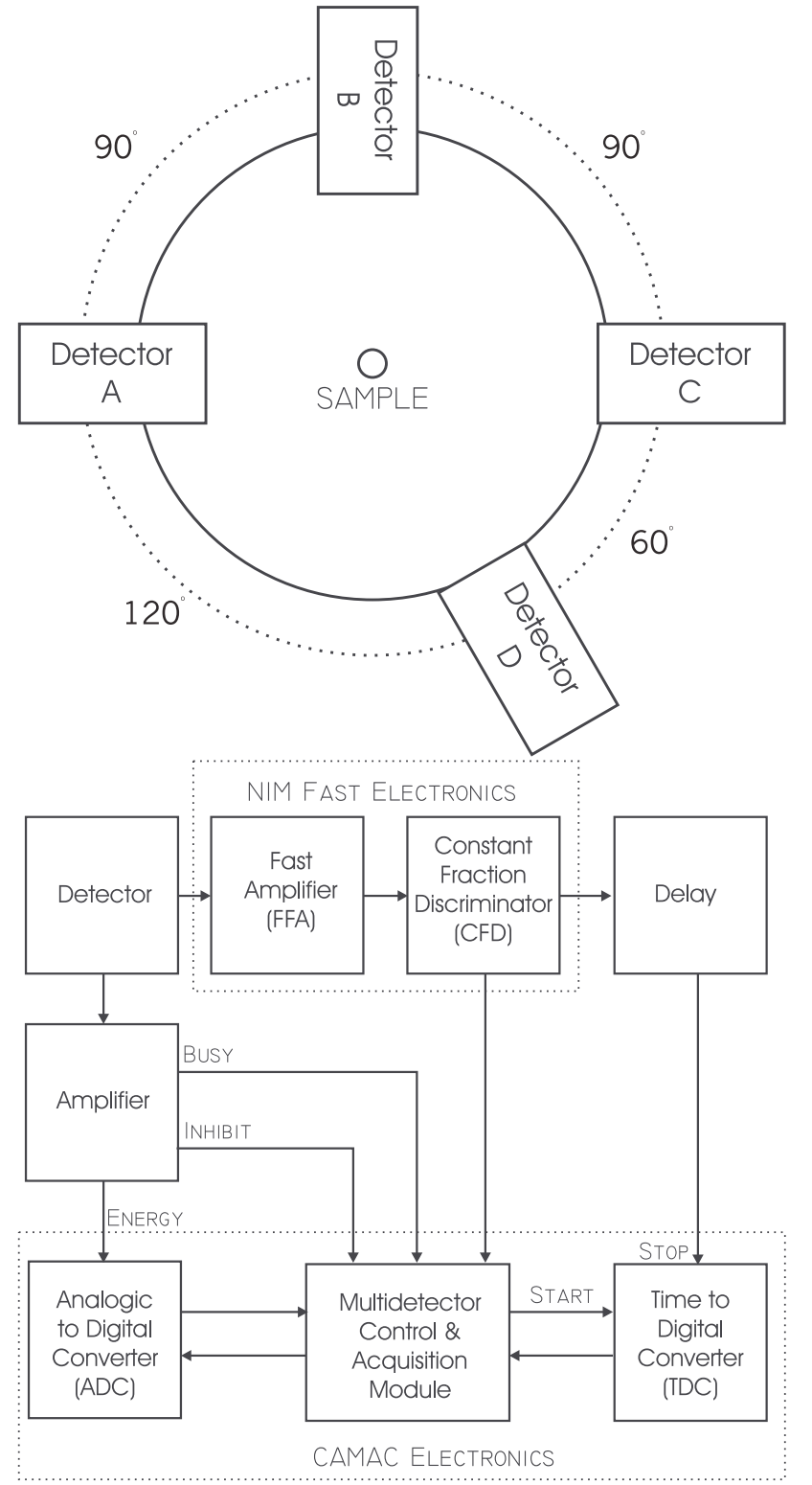

Fig. 1. Top: Schematics of the planar detector setup used in the present measurements; Bottom: Electronical setup used in the present experiment.

special care was taken as to fit only one of the mixing ratios on a cascade, using the tabulated value (or the result of a previous fit using other cascade) for the other, in order to avoid the problems described in [8]. Also, both "sides" of a cascade (i.e., $\gamma_{1} \times \gamma_{2}$ and $\left.\gamma_{2} \times \gamma_{1}\right)$ were fitted together.

\subsection{Angular correlation plots}

One additional problem in this type of analysis is the matter of the graphical representation of the results. As the $Q_{k k}$ coefficients affect non-linearly the angular correlation function $W(\theta)$ (eq. (1)) and only make sense for real, finite detectors, the fitted angular correlation function can only be directly compared to the experimental results at the angles where real 

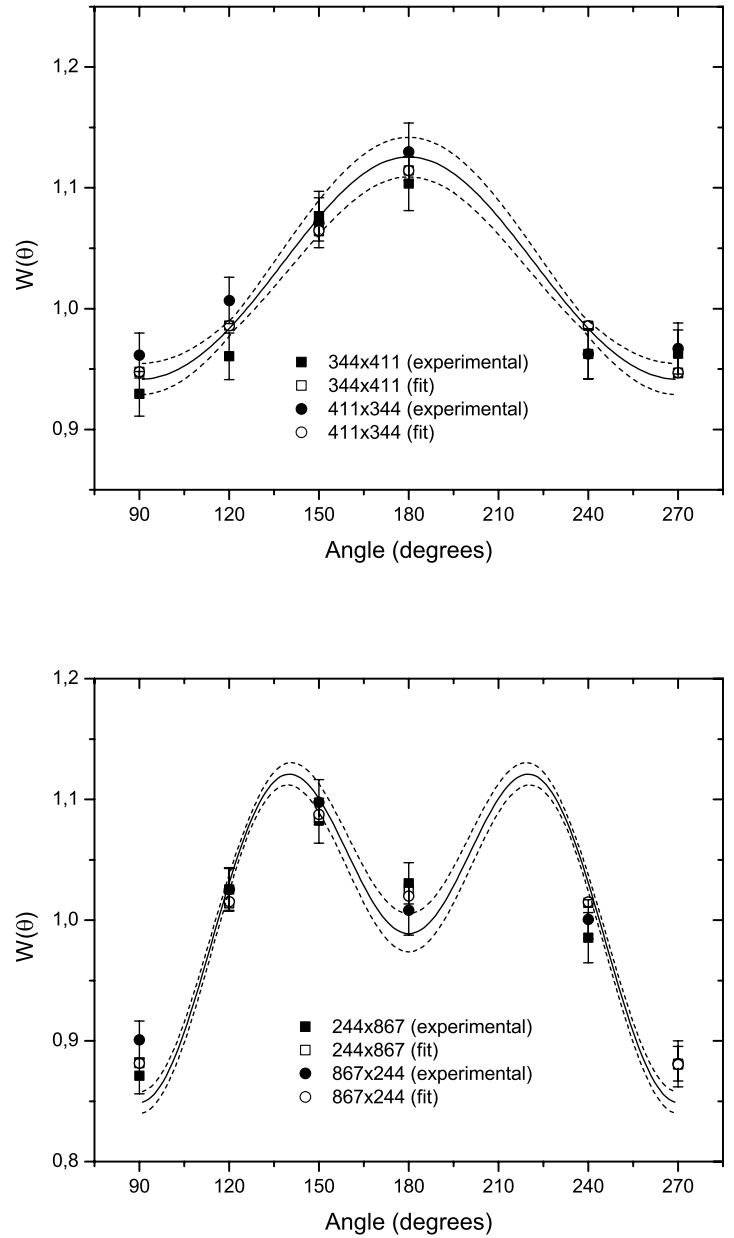

Fig. 2. Experimental results for the $344 \mathrm{keV} \times 411 \mathrm{keV}$ (top) and $867 \mathrm{keV} \times 244 \mathrm{keV}$ (bottom) cascades from ${ }^{152} \mathrm{Eu}$.

detectors exist, so that the correction can be applied to the calculated values; as a consequence, it's not possible to draw a continuous curve for $W(\theta)$ that can be compared to the experimental results. A suggestion to overcome this is shown in figure 2, where the experimental data is plotted together with the fitted results (corrected for the real detectors pairs) and the continuous $W(\theta)$ curve which was not corrected; together with that, the graph shows the confidence bands calculated as the band covered within $1 \sigma$ intervals for each of the fit parameters $(\alpha$ and $\delta$ ).

\section{Results and discussion}

Overall, the multipolar mixing ratios for 10 transitions were evaluated: the $1332.5 \mathrm{keV}$ transition from the decay of ${ }^{60} \mathrm{Co}$, the 1213.0 and $867.4 \mathrm{keV}$ transitions from the electron capture decay of ${ }^{152} \mathrm{Eu}$, the 411.1 and $778.9 \mathrm{keV}$ transitions from the $\beta^{-}$decay of ${ }^{152} \mathrm{Eu}$ and the $460.5,107.1,321.6,361.8$ and $251.6 \mathrm{keV}$ transitions from the $\beta^{-}$decay of ${ }^{193}$ Os (the ${ }^{193} \mathrm{Os}$ decay data are part of a larger work, which can be found in $[9,10])$. The results of the multipolar mixing ratio $(\delta)$ for these transitions are compared to the reference values (found in [5] for the standard sources and in [6] for the ${ }^{193}$ Os decay)
Table 1. Experimental values for some transitions with welldetermined mixing ratios $(\delta)$ compared to the compiled values from [6] (for ${ }^{193} \mathrm{Os}$ ) and [5].

\begin{tabular}{llll}
\hline Nuclide & $\begin{array}{l}\text { Transition } \\
(\mathrm{keV})\end{array}$ & $\begin{array}{l}\delta \\
\text { (this work) }\end{array}$ & $\begin{array}{l}\delta \\
\text { (refs. [5,6]) }\end{array}$ \\
\hline${ }^{60} \mathrm{Co}$ & 1332.5 & $+0.001(15)$ & 0 \\
${ }^{152} \mathrm{Eu}$ & 1213.0 & $-0.007(17)$ & $0.00(2)$ \\
& 867.4 & $-5.3(4)$ & $-6.5(3)$ \\
& 411.1 & $+0.032(22)$ & 0 \\
& 778.9 & $+0.026(10)$ & $+0.002(6)$ \\
${ }^{193} \mathrm{Os}$ & 460.5 & $-0.634(17)$ & $-0.64(3)$ \\
& 107.1 & $+0.171(13)$ & $+0.164(8)$ \\
& 321.6 & $+0.236(17)$ & $+0.234(10)$ \\
& 361.8 & $-0.314(27)$ & $-0.33(3)$ \\
& 251.6 & $-0.132(7)$ & $-0.079(20)$ \\
\hline
\end{tabular}

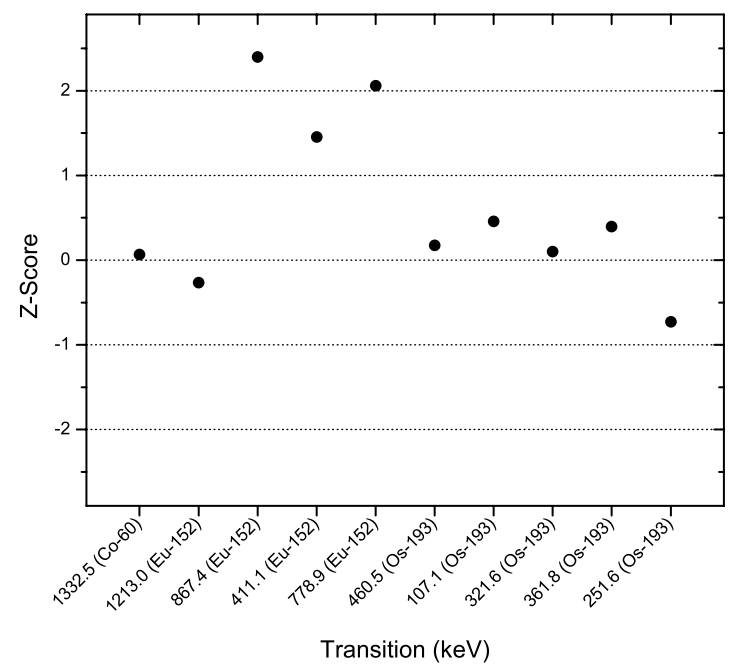

Fig. 3. Z-Score comparison of the multipolar mixing ratio values found in this work to the tabulated values [5,6] for each of the transitions shown in table 1 .

in table 1; the Z-Score for the comparison of the present values and the reference ones are shown in figure 3. This comparison shows that the results found in the present work are basically compatible to the reference values, with the exception of the 887.4 and $778.9 \mathrm{keV}$ transitions from the ${ }^{152} \mathrm{Eu}$ source; in the first case, our results agree to the fact that the $867.4 \mathrm{keV}$ transition is a strongly-mixed E2+M1 transition and also agrees on the sign of the mixing ratio, but the resulting $\mathrm{Z}$-Score is more than two with our results indicating a smaller M1 contribution than the reference value; in the second case, the $778.9 \mathrm{keV}$ transition is expected to be a E1 transition, but our results show a distinct M2 contribution.

\section{Conclusions}

The methodology proposed in this work was applied to the determination of the multipolar mixing ratios for 10 transitions from both ${ }^{60} \mathrm{Co}$ and ${ }^{152} \mathrm{Eu}$ standard sources and from the $\beta^{-}$decay of ${ }^{193}$ Os. The results obtained using the proposed 
methodology were mostly compatible with the ones found in the literature, with only two of the values differing significantly (Z-Score $>2$ ) from the compiled ones.

The authors would like to thank the staff in the IFUSP Linear Accelerator Laboratory (LAL-IFUSP) for the use of the multiparametric data acquisition system.

\section{References}

1. H. Frauenfelder, R.M. Steffen, in Alpha-, Beta- and GammaRay Spectroscopy, Vol. 2, edited by K. Siegbahn (North-Holland, Amsterdam, 1965), pp. 997-1198.

2. M.J.L. Yates, in Alpha-, Beta- and Gamma-Ray Spectroscopy, Vol. 2, edited by K. Siegbahn (North-Holland, Amsterdam, 1965), pp. 1691-1703.

3. D.R. Camp, A.L. Van Lehn, Nucl. Instrum. Meth. 76, 192 (1969).
4. M. Ferentz, N. Rosenzweig, in Alpha-, Beta- and Gamma-Ray Spectroscopy, Vol. 2, edited by K. Siegbahn (North-Holland, Amsterdam, 1965), pp. 1687-1690.

5. R.B. Firestone, V.S. Shirley, C.M. Baglin, S.F. Chu, J. Zipkin, Table of Isotopes, 8th edn. (John Wiley and Sons, New York, 1996).

6. E. Achterberg, O.A. Capurro, G.V. Marti, V.R. Vanin, R.M. Castro, Nucl. Data Sheets 107, 1 (2006).

7. J.Y.Z. Chávez, F.A. Genezini, M.T.F. da Cruz, C.B. Zamboni, M.N. Martins, V. Vanin, Z.O. Guimarães Filho, P.R. Pascholati, Rev. Sci. Instrum. 76, 1 (2005).

8. R.A.A.M. Oliveira, Master's thesis, Instituto de Física da Universidade de São Paulo, São Paulo (1986).

9. G.S. Zahn, C.B. Zamboni, F.A. Genezini, J.Y. Zevallos-Chávez, M.T.F. da Cruz, Angular correlation study of excited levels in ${ }^{193}$ Ir , in AIP Conference Proceedings 884: VI Latin American Symposium on Nuclear Physics and Applications (American Institute of Physics, New York, 2005), pp. 442-445.

10. G.S. Zahn, Ph.D. thesis, Instituto de Pesquisas Energéticas e Nucleares (2006). 